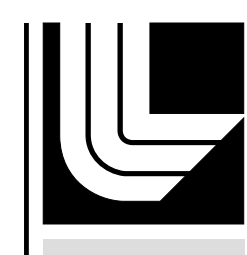

Fabrication of Mo/Cu Multilayer and Bilayer Transition Edge Sensors

Z. A. Ali, O. B. Drury, M. F. Cunningham, J. M. Chesser, T. W. Barbee Jr., S. Friedrich

October 6, 2004

Harnessing The Magic ASC/04 Jacksonville, FL, United States October 3, 2004 through October 8, 2004 
This document was prepared as an account of work sponsored by an agency of the United States Government. Neither the United States Government nor the University of California nor any of their employees, makes any warranty, express or implied, or assumes any legal liability or responsibility for the accuracy, completeness, or usefulness of any information, apparatus, product, or process disclosed, or represents that its use would not infringe privately owned rights. Reference herein to any specific commercial product, process, or service by trade name, trademark, manufacturer, or otherwise, does not necessarily constitute or imply its endorsement, recommendation, or favoring by the United States Government or the University of California. The views and opinions of authors expressed herein do not necessarily state or reflect those of the United States Government or the University of California, and shall not be used for advertising or product endorsement purposes. 


\title{
Fabrication of $\mathrm{Mo} / \mathrm{Cu}$ Multilayer and Bilayer Transition Edge Sensors
}

\author{
Z. A. Ali, O. B. Drury, M. F. Cunningham, J. M. Chesser, T. W. Barbee Jr., S. Friedrich
}

\begin{abstract}
We are developing cryogenic high-resolution $\mathrm{x}$-ray, $\gamma$-ray and neutron spectrometers based on superconducting $\mathrm{Mo} / \mathrm{Cu}$ transition edge sensors. Here we discuss the sensor design for different applications, present the photolithographic fabrication techniques, and outline future detector development to increase spectrometer sensitivity.
\end{abstract}

Index Terms - Superconducting transition edge sensors, device fabrication, multilayers, X-ray spectrometers, Gamma-ray spectrometers.

\section{INTRODUCTION}

$\mathrm{S}_{\mathrm{s} u n}^{\mathrm{u}}$ UPERCONDUCTING transition edge sensors (TES) are superconducting thermistors operated at the transition from the superconducting to the normal state and weakly coupled to a cold bath. They rely on measuring the increase in temperature after photon or particle absorption, and can be adapted for detection of X-rays, $\gamma$-rays, and neutrons in high resolution cryogenic spectrometers. Over the last decade, they have been developed for applications ranging from highenergy astrophysics to nuclear science and biophysics [1]. In the simplest case, the energy resolution of these devices is limited by $\Delta \mathrm{E}_{\mathrm{FWHM}} \approx 2.355\left(\mathrm{k}_{\mathrm{B}} \mathrm{T}^{2} \mathrm{C}\right)^{1 / 2}$, where $\mathrm{T}$ is the operating temperature and $\mathrm{C}$ is the device heat capacity. High-resolution spectroscopy therefore requires low operating temperatures, with $\mathrm{T} \approx 0.1 \mathrm{~K}$ being a good compromise between good energy resolution and ease of operatio. In addition, high sensitivity, typically specified in terms of the dimensionless parameter $\alpha \equiv \mathrm{T} / \mathrm{R} \cdot \partial \mathrm{R} / \partial \mathrm{T}$, requires the transition between the superconducting and normal state to be sharp, and device linearity requires the transition to be smooth. Finally, device reproducibility, scalability to large arrays, and long-term stability are necessary. Here we discuss the fabrication process we have developed to achieve these goals.

\section{THEORY}

\section{A. Design Consideratios}

To fabricate devices with the appropriate $\mathrm{T}_{\mathrm{C}}$ we exploit the

Manuscript received October 3, 2004. Funding was provided by the US Department of Energy, NA-22, under grant LL0035-DP. This work was preformed under the auspices of the U.S. Department of Energy by Lawrence Livermore National Laboratory (UC LLNL) under contract No. W-7405-ENG-48.

The authors are with the Lawrence Livermore National Laboratory, L-270, Livermore, CA 94750, USA (Corresponding author: S. Friedrich: 925-4231527; fax: 925-424-5512; e-mail: friedrich1@1lnl.gov). proximity effect, i.e. the suppression of superconductivity in a superconducting material in proximity with a normal material over a characteristic coherence length of $\sim 100 \mathrm{~nm}[2,3]$. The thickness ratio of the superconducting $\left(\mathrm{d}_{\mathrm{sc}}\right)$ and the normal metal layer $\left(\mathrm{d}_{\mathrm{n}}\right)$ governs the $\mathrm{T}_{\mathrm{C}}$ of the device. The proximity of two such metals causes concerns for intermetallic diffusion and thus for the long-terms stability of the TES thermistors. We therefore choose Mo as the superconductor and $\mathrm{Cu}$ as the normal metal, two materials that do not form intermetallic phases at room temperature. The desire for high sensitivity (large $\partial \mathrm{R} / \partial \mathrm{T}$ ) and linear response (smooth transitions) demands good control of the $\mathrm{d}_{\mathrm{sc}} / \mathrm{d}_{\mathrm{n}}=\mathrm{d}_{\mathrm{Mo}} / \mathrm{d}_{\mathrm{Cu}}$. Normal metal banks create normal boundary conditions, which have been shown to increase the sensitivity $\alpha$ and the device linearity in superconducting bilayers [4].

High energy resolution requires a homogenous detector response, i.e. no spatial variation of the response over the detector area. This requires the thermalization time $\left(\tau_{\text {therm }}\right)$ over which the photon energy is distributed homogeneously throughout the detector to be much shorter than the pulse rise time, which is typically set by the speed of the readout electronics $\left(\tau_{\text {elec }}\right)$. This leads to two cases. If the TES serves as both absorber and sensor, as is the case with optical, UV, and $\mathrm{X}$-ray detectors, then the TES itself must have high thermal conductivity, which in turn demands high electrical conductivity and low device resistivity. If the absorber is separate from the detector, as in the case of $\gamma$-ray and neutron detectors, the absorber must have fast thermalization, allowing the TES to be optimized according to other consideratios, such as a particular desired device resistance.

\section{B. Bilayer Transition Edge Sensors}

Bilayers consist of one superconducting film and one normal metal film, typically deposited immediately on top to ensure good coupling. The normal metal can be made comparatively thick, of order several $100 \mathrm{~nm}$, subject to the constraint of the finite coherence length of the proximity effect. For high purity metals, the electron mean free path is fundamentally set only by boundary scattering, leading to low resistivity and fast thermalization. For a TES with an area $\mathrm{L}^{2}$, a thermalization time

$$
\tau_{\text {therm }}=\frac{L^{2}}{D} \text { where } D=\frac{1}{e^{2} n\left(E_{F}\right) \rho}
$$

shorter than $\tau_{\text {elec }} \approx 1 \mu$ s requires a high electrical resistivity. In 
the case of $\mathrm{Cu}\left(\rho_{\mathrm{Cu}, 300 \mathrm{~K}}=1.7 \mu \Omega \mathrm{cm}\right)$, this leads to the requirement of a residual resistance ratio, $R R R=R_{300 K} / R_{4.2 K}$, a measure of the purity of the film, of $\sim 10$. We choose a thickness of the $\mathrm{Cu}$ film of $\mathrm{d}_{\mathrm{Cu}}=200 \mathrm{~nm}$ and adjust the critical temperature by adjusting the thickness of the Mo film, $\mathrm{d}_{\mathrm{Mo}}$.

The critical temperature of a Mo/Cu bilayer with $\mathrm{d}_{\mathrm{Cu}}=200$ $\mathrm{nm}$ can be determined according to $[5,6]$

$$
T_{C}=T_{C O}\left[\frac{d_{M o}}{d_{o}} \frac{1}{(1.13(1+1 / v)) t}\right]
$$

where $v=d_{C u} n_{C u} / d_{M o} n_{M o}$ and $1 / d_{o}=(\pi / 2) k_{B} T_{C O} \lambda_{F}^{2} n_{M o}$.

Here $\mathrm{T}_{\mathrm{CO}}$ is the bulk critical temperature of the superconducting material, $\lambda_{\mathrm{F}}$ is the Fermi wavelength, and transmissivity, $t$, is a factor introduced to characterize the quality of the coupling between the superconducting and the normal metal layer. Figure 1 shows the theoretical critical temperature according to (2) as a function of Mo thickness for a $\mathrm{Cu}$ thickness of $200 \mathrm{~nm}, \mathrm{~d}_{\mathrm{o}}=1.18 \mu \mathrm{m}, \mathrm{n}_{\mathrm{Cu}} / \mathrm{n}_{\mathrm{Mo}}=0.431, \lambda_{\mathrm{F}}=$ $0.462 \mathrm{~nm}$, and $\mathrm{T}_{\mathrm{CO}}=1.01 \mathrm{~K}$ [6]. The different curves correspond to different degrees of coupling between the two layers, with typical experimental values between $0.1 \leq \mathrm{t} \leq 0.4$.

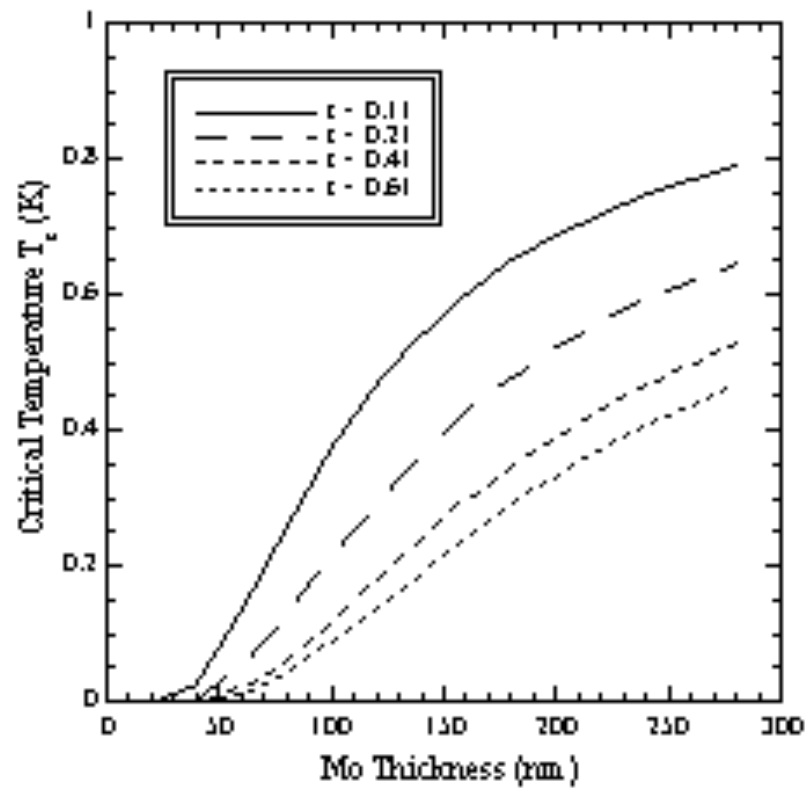

Fig. 1: Predicted bilayer $\mathrm{T}_{\mathrm{C}}$ vs. Mo thickness with the $\mathrm{Cu}$ film thickness set to $200 \mathrm{~nm}$ for different values of the transmissivity t. Improved coupling between the two films, i.e. higher $t$, increases the influence of the normalconducting $\mathrm{Cu}$ film and therefore reduces $\mathrm{Tc}$.

\section{Multilayer Transition Edge Sensors}

The multilayer TES is a superlattice [7] of alternating thin layers of a superconductor and a normal metal. As in the case of bilayers the ratio of superconducting and the normal metal sets the critical temperature, but the maximum thickness is no longer constrained by the coherence length. The TES can therefore be made arbitrarily thick and its resistance and current density be adjusted accordingly without changing the $\mathrm{T}_{\mathrm{C}}$. This is an advantage because high current densities are known to reduce the device sensitivity $\alpha$. Furthermore, thickness variations at the edges turn out to be less crucial, and multilayers do not require any normal metal banks to ensure high sensitivity. On the other hand, the thin individual layers cause the electron mean free path to be short, leading to a low diffusion constant and slow thermalization within the multilayer TES. Multilayer TESs can therefore only be used in composite $\gamma$ or neutron detectors in which the energy thermalization occurs in the attached absorber [8,9].

\section{TES FABRICATION}

We fabricate TES devices on 4" Single Side Polished, ptype Si [100] wafers purchased from Temic Semiconductor. $500 \mathrm{~nm}$ silicon nitride ( $\mathrm{SiN})$ are thermally grown on the wafers via low-pressure chemical-vapor-deposition (LPCVD), with a compressive stress of $785 \mathrm{MPa}$. The wafer is baked for $15 \mathrm{~min}$ at $120^{\circ} \mathrm{C}$ to remove surface contamination. Immediately before coating the wafer with AZ1518 (positive) photoresist (PR) it is soaked in hexamethyldesalizane (HMDS) for $3 \mathrm{~min}$ to remove surface moisture and increase PR adhesion. The rough side is coated first followed by the polished side. After a 10 min soft bake at $90^{\circ} \mathrm{C}$, a second $\mathrm{PR}$ coat is applied to the rough side, followed by another 10 minute bake at $90^{\circ} \mathrm{C}$. Both sides of the wafer are exposed for $7.5 \mathrm{~s} \times 40 \mathrm{~mW} / \mathrm{cm}^{2}$ in a back-side aligner to define alignment marks, reactively etched to a depth of $150 \mathrm{~nm}$ with $200 \mathrm{mTorr}$ of $\mathrm{CF}_{4}+10 \% \mathrm{O}_{2}$ at $150 \mathrm{~W}$ for $30 \mathrm{~s}$. Finally, the rough side is etched in $45 \% \mathrm{KOH}$ at $65^{\circ}$ at $16 \mu \mathrm{m}$ /hour until only the $\mathrm{SiN}$ windows remain that will form the weak thermal link between the TES and the cold bath.

Before depositing the bilayer or multilayer sensor, the wafers are UV-cleaned to remove any hydrocarbon buildup on the SiN. The multilayers are deposited in a UHV sputtering system originally designed for multilayer X-ray optics. It has a base pressure of $10^{-9}$ Torr and is baked out before each production run. The SiN-coated Si wafers are mounted on a rotating arm opposite two horizontally mounted DC magnetron sputtering targets with $99.9995 \%$ purity. Baffling inside the chamber is used to prevent cross contamination. During deposition the sputtering pressure is maintained at 2.5 mTorr of $99.999 \%$ Ar gas. The thickness of the films depends on sputter power and the rate at which the wafers are rotated over the targets. The Mo is sputtered first because it adheres to the $\mathrm{SiN}$ better than $\mathrm{Cu}$. This system reliably produces $\mathrm{Mo} / \mathrm{Cu}$ multilayers with an interfacial surface roughness of $<1 \AA$.

The bilayers are deposited in a different sputtering system with a base pressure of $10^{-8}$ Torr. Because this system has an isolated load lock, it does not need to be baked out before each run, but is continuously maintained at $10^{-8}$ Torr. The wafers are held stationary above vertically mounted 2 " DC magnetron sputtering targets $99.999 \%$ pure. Again, baffling prevents cross contamination, and the film thickness depends on the sputtering time and power. 


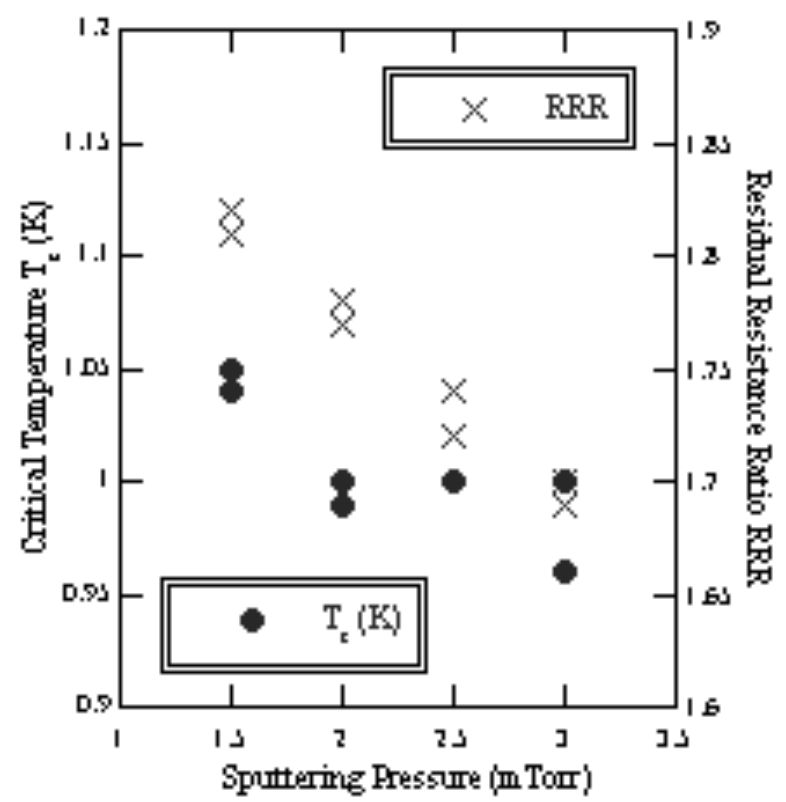

Fig. 2 : Critical temperature and film purity (RRR) as a function of sputtering pressure. Experiments have been repeated to test reproducibility.

To optimize the superconducting properties of our Mo films we have investigated the effects of sputtering pressure on Tc and RRR, which is known to affect film purity [10] and stress [11] (figure 2). A slightly compressive Mo film is desirable for a critical temperature close to the bulk value of $1.01 \mathrm{~K}$. We find that $T_{C}$ does vary in the range of preferable deposition pressures. As expected, a higher sputtering pressure also reduces the residual resistivity ratio RRR because more sputtering atoms are incorporated into the Mo film. While a pressure of $1 \mathrm{mTorr}$ at a typical puttering power of $125 \mathrm{~W}$ produces films with the highest $\mathrm{Tc}$, we typically deposit at 2.5 mTorr, because the plasma needed for sputtering fails sporadically below $2.5 \mathrm{mTorr}$ Ar deposition pressure.

To fabricate bilayer TESs, we first selectively remove the $\mathrm{Cu}$ top layer, leaving in only in the area of the TES and the wiring, using a commercial Transene APS $\mathrm{Cu}$ etchant based on ammonium persulfate for $\sim 20 \mathrm{~s}$. We subsequently etch the Mo only for $\sim 7 \mathrm{~s}$ in the same area, using Transene's proprietary Mo Etchant Type TFM based on sodium hydroxide/potassium ferrocyanide. The exact compositions of these etchants are proprietary to Transene Corp. This does not attack the $\mathrm{Cu}$ overlayer and thus leaves a $\mathrm{Cu}$ overhang over the Mo base layer that provides normal metal boundary conditions. Finally, the area of the TES is defined with a second $\sim 20 \mathrm{~s} \mathrm{Cu}$ etch that removes the $\mathrm{Cu}$ from the wiring area. This also exposes the area of the Mo bonding pads. Throughout our photolithographic process, we use AZ1518 positive $\mathrm{PR}$ and AZ 1:1 developer and soak the wafer in HDMS before applying PR to eliminate moisture. While this process has produced bilayers with the appropriate critical temperature and sensitivity, it is at present not sufficiently reproducible to reliably fabricate TES arrays with sufficient homogeneity.

The multilayer TES fabrication is more involved. First, the area of the TES is defined by etching through both $\mathrm{Mo}$ and $\mathrm{Cu}$ at once using a $1: 2: 2$ solution of $\mathrm{HF}: \mathrm{HNO} 3: \mathrm{H}_{2} \mathrm{O}$ for $2 \mathrm{~s}$. This is a very aggressive etch, and is typically chilled overnight to reduce the etch rate. After each etching process we rinse the wafers in de-ionized water and then blow them dry with $\mathrm{N}_{2}$, and after each process that involves PR we clean the wafers with acetone and methanol. Then we pattern a wiring layer and deposit a layer of $300 \mathrm{~nm}$ Mo. We define the wiring layer by lift-off in which the unwanted Mo is removed by the PR underneath it. After deposition, the wafer is agitated in acetone, until the unwanted Mo is removed. Each $10 \mathrm{~cm}$ wafer has many devices on it. To test single devices the wafer is coated with an extra thick layer of PR and then diced by a diamond saw after which the PR is removed and we can use each chip.

\section{Results}

We have measured the dependence of the $T_{C}$ of the multilayer TES devices as a function of the $\mathrm{Mo} / \mathrm{Cu}$ ratio. The Mo thickness was held constant at $2.0 \mathrm{~nm}$ while the $\mathrm{Cu}$ was varied from $1.0 \mathrm{~nm}$ to $7.0 \mathrm{~nm}$. These films alternated to have a total thickness of $200 \mathrm{~nm}$ (figure 3). As expected, the Tc decreases with increasing $\mathrm{Cu}$ thickness, and a ratio of $2 \mathrm{~nm}$ $\mathrm{Mo}$ and $7 \mathrm{~nm} \mathrm{Cu}$ produces films of the desired Tc. An exponential fit to the data agrees with the Cooper prediction $[3]$ in the limit of thin films.

We have also measured the variation in $\mathrm{T}_{\mathrm{C}}$ as a function of the thickness of the multilayer for a constant $\mathrm{Mo}: \mathrm{Cu}$ ratio of $2: 7$. It confirms that the critical temperature of the multilayer does not change with its overall thickness as long as the Mo : $\mathrm{Cu}$ ratio is kept constant (inset figure 3). This allows us to adjust current density and device resistance independently of Tc, and ensures that the sensitivity is not reduced due to overly high current densities.

Note that the 2" sputtering targets used to deposit the multilayers on our 4" wafers produce a spatially varying profile over the length of the wafer. This produces spatial

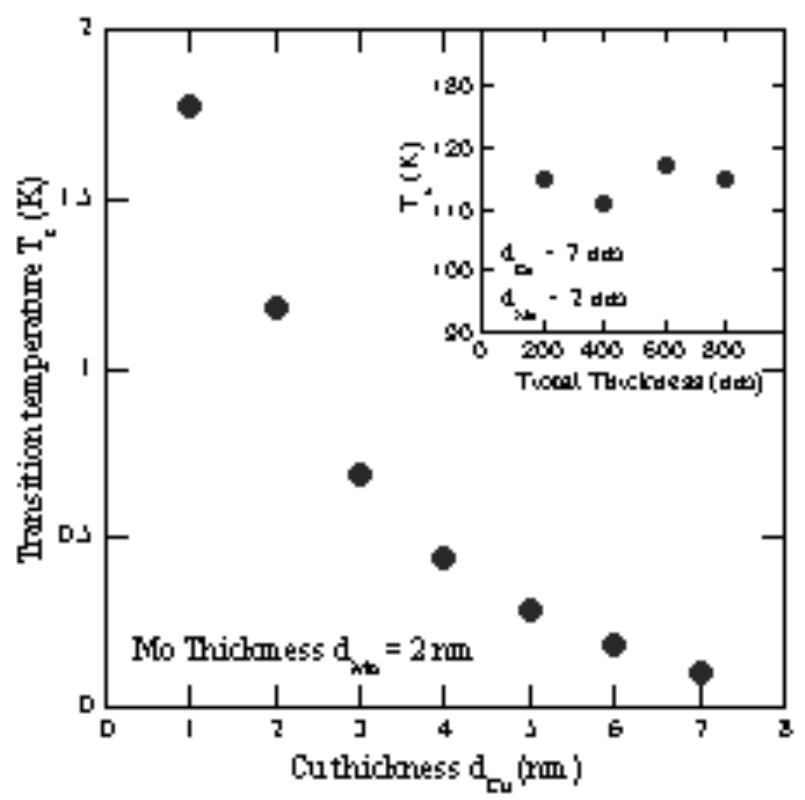

Fig. 3: $\mathrm{T}_{\mathrm{C}}$ vs. $\mathrm{Cu}$ thickness per layer with Mo thickness held at $2 \mathrm{~nm}$ per layer. (Inset) $\mathrm{T}_{\mathrm{C}}$ vs. Total TES thickness (mo/Cu ratio is $2 / 7$ ) shows no dependence. 


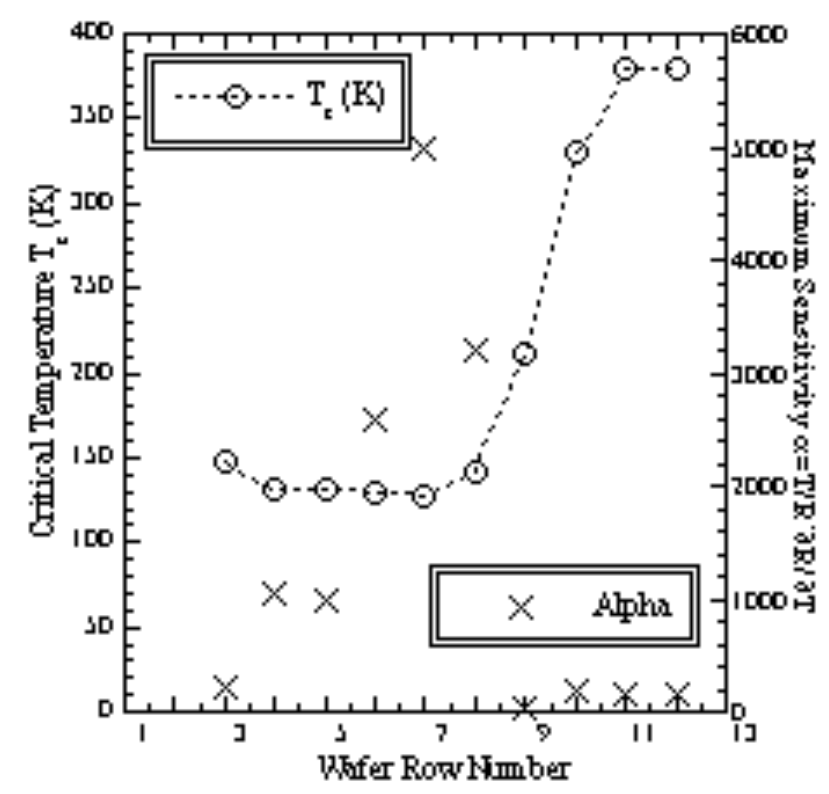

Fig. 4: Spatial variations of the critical temperature and the sensitivity across the 4" area of our wafer.

variations of the critical temperature Tc and the sensitivity a (figure 4). However, over a $\sim 1.5$ " wide area of the wafer where it directly faces the sputtering targets, the $\mathrm{Tc}$ is reproducible and the sensitivity exceeds 1000 in the unbiased case. During operatio, the finite bias current through the TES reduces this value to $100-200$, which is sufficient for all applications we pursue.

We adapt the multilayer for specific applications with the appropriate choice of absorber. For X-rays, we deposit $500 \mathrm{~nm}$ of $\mathrm{Au}$ by $\mathrm{DC}$ magnetron sputtering and pattern it by photolithographic lift off. Au has low resistance and ensures fast thermalization. For $\gamma$-ray detection we attach $250 \mu \mathrm{m}$ thick Sn foils with an area varying between $1 \times 1$ and $2 \times 2 \mathrm{~mm}$. Tin combines moderately high detection efficiency and low heat capacity, and quickly thermalizes the absorbed energy without resolution degradation [8]. It is glued on with Stycast thermal epoxy using Al spacers underneath to ensure that the absorber does not touch the SiN membrane. The epoxy also serves as a bottle neck for the incident energy, thus assuring that $\tau_{\text {elec }}>\tau_{\text {therm }}$ [12]. Such devices have shown a very high resolving power $\mathrm{E} / \Delta \mathrm{E} \geq 1000$ in the energy range up to 100 $\mathrm{keV}$ [8]. For neutron detection, we use a material with a high neutron absorption cross section, large Q-value and simple response function, such as provided by ${ }^{6} \mathrm{Li}$ or ${ }^{10} \mathrm{~B}$ in $\mathrm{LiF}$ or $\mathrm{TiB}_{2}$ crystals $[9,14]$. Initial attempts to deposit $\mathrm{Mo} / \mathrm{Cu}$ bilayers directly onto the LiF crystal had to be abandoned because of the high surface roughness of the LiF compared to the $\mathrm{SiN}$ membranes (figure 5). Instead, we attach a TES on a small $\mathrm{Si}$ chip to the crystal with GE varnish. Such neutron absorbers show an energy resolution of $46 \mathrm{keV}$ for a total energy of 4.7 $\mathrm{MeV}$ despoited in LiF by themal neutrons [14].
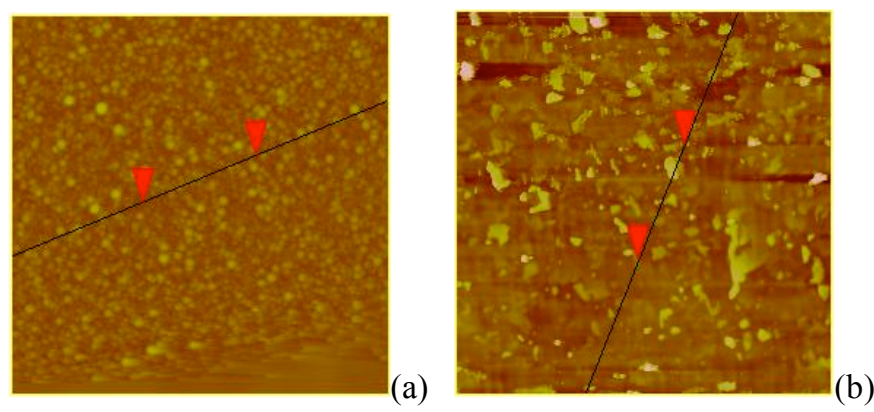

(b)

Fig. 6: (a) Profile of SiN substrate taken with an atomic force microscope (AFM) shows a roughness of $5 \mathrm{~nm}$ rms. (b) Profile of a LiF absorber taken with an AFM shows the polycrystalline structure of the LiF crystal and a roughness of $100 \mathrm{~nm}$ RMS. The surface roughness increases after etching because of anisotropic etching along different crystal planes.

\section{SUMMARY}

We have developed a robust multilayer full-wafer fabrication process to manufacture single pixels and arrays of $\mathrm{Mo} / \mathrm{Cu}$-based TES thermistors. Our process is easily adaptable to produce $\mathrm{x}$-ray, $\gamma$-ray, and neutron detectors. Our Gammaray detectors based on multilayers with $\mathrm{Sn}$ absorbers have produced high energy resolution below $100 \mathrm{eV}$ FWHM for photon energies below $100 \mathrm{keV}$, and we have started to fabricate small arrays. The multilayers TESs performance is also sufficient for high-resolution fast neutron spectrometry. For X-ray detection, multilayers have too high internal resistance, and we are developing $\mathrm{Mo} / \mathrm{Cu}$ bilayer TESs for improved themalization.

\section{REFERENCES}

[1] A good overview of the field of cryogenic detectors is given in the proceedings of the $10^{\text {th }}$ International Conference on Low Temperature detectors, LTD-10, published as Nucl. Inst. Meth., vol. A520 (2004).

[2] P. G. de Gennes, "Boudary Effects in Superconductors," Rev. Mod. Phys., pp. 225-237 (1964).

[3] L. Cooper, Phys Rev. Lett., vol. 6, pp.698-700 (1961).

[4] Hilton et al., "Microfabricated Transition-Edge X-Ray Detectors," IEEE Conf. Appl. Super. 11(1), pp.739-742 (2001).

[5] K.D. Usadel, Phys. Rev. Lett., vol. 25, pp. 507-509 (1970)

[6] J. M. Martinis, "Calculation of $\mathrm{T}_{\mathrm{C}}$ in a normal-superconductor bilayer using the microscopic-based Usadel theory," Nuc. Meth. Inst. A, vol. 444 (2000) pp..23-27.

[7] B.Y. Jin \& J.B. Ketterson, “Artificial Superlattices," Adv. in Phy., vol. 38, No. 3 (1989), pp. 189-366.

[8] S. Friedrich et al., "Design of a multi-channel ultra-high resolution gamma-ray superconducting spectrometer," SPIE Conf. Proc.

[9] T. Niedermayr et al., "Microcalorimeter design for a fast-neutron spectrometer," Nuc. Meth. Inst. A, vol 520, (2004).

[10] L.P. Kendig et al., "The role of impurities and microstructure on residual stress in nanoscale Mo films," Surface and Coatings Technology, Vol. 134 (2000), pp. 124-129.

[11] Hoffman \& Thornton, "Internal stresses in Cr, Mo, Ta, and Pt films deposited from a planar magnetron source," J. Vac. Sci. Technology, No. 20 Vol. 3 (1982), pp. 355-358.

[12] M.L. van de Berg et al., "High resolution hard X-ray and gamma-ray spectrometers based on superconducting absorbers coupled to superconducting transition edge sensors," Proc. SPIE 4140 (2000).

[13] M.F. Cunningham, Thesis University of California Davis (2002), pp.4550.

[14] Z. Bell et al, "Neutron Detection with Cryogenics and Semiconducters," accepted for publication in Phys. Sol. (2004). 